\title{
Infant exposure to persistent organochlorine compounds is higher in Denmark than in Finland
}

\author{
H. Shen ${ }^{1,2}$, K. M. Main ${ }^{2}$, A.-M. Andersson ${ }^{2}$, I. N. Damgaard ${ }^{2}$, \\ H. E. Virtanen ${ }^{3}$, N. E. Skakkebaek ${ }^{2}$, J. Toppari ${ }^{3}$ \\ \& K.-W. Schramm ${ }^{1,4}$ \\ ${ }^{1}$ Institute of Ecological Chemistry, Helmholtz Zentrum München - \\ German Research Center for Environmental Health, \\ Ingolstädter Landstrasse 1, D-85764, Neuherberg, Germany \\ ${ }^{2}$ University Department of Growth \& Reproduction, Rigshospitalet, \\ Blegdamsvej 9, DK-2100, Copenhagen, Denmark \\ ${ }^{3}$ Departments of Physiology and Paediatrics, University of Turku, \\ Kiinamyllynkatu 10, FI-20520, Turku, Finland \\ ${ }^{4}$ Technische Universität München, \\ Department für Biowissenschaftliche Grundlagen, \\ Weihenstephaner Steig 23, Freising, Germany
}

\section{Abstract}

Significantly poorer male reproductive health has been documented in Danish compared to Finnish men, including a higher prevalence of cryptorchidism and hypospadias. Exposure to environmental pollutants with endocrine disrupting activities has been suggested as a possible contributing factor. In this study, we investigated whether there was a difference in milk and placenta concentrations of persistent organohalogen compounds between the two countries. Organohalogens were analyzed by high-resolution gas chromatography-high resolution mass spectrometry in human milk samples from Finland $(n=65)$ and Denmark $(\mathrm{n}=65)$ and placentas $(\mathrm{n}=168$ Danish, 112 Finnish), selected from a population-based cohort. $p$, $p$ '-DDE was the dominant pollutant. $\beta-\mathrm{HCH}, \mathrm{HCB}$, END-I, dieldrin, OXC, $c$-HE, and $p, p^{\prime}$-DDT were the other main organochlorines detected. Danish samples had significantly higher concentrations of $p, p^{\prime}$-DDE, $p, p$ '-DDT, $\beta-\mathrm{HCH}, \mathrm{HCB}$, dieldrin, c-HE, and OXC than Finnish. The organochlorine levels were higher in Danish samples, which suggests higher exposure of Danish infants.

Keywords: placenta, breast milk, organochlorine pesticides, organobromine compounds, infants. 


\section{Introduction}

Danish men have a higher prevalence of testicular cancer and poor semen quality compared to Finnish men [1,2], and there is a higher prevalence of cryptorchidism and hypospadias among Danish newborn boys compared to Finnish [3-5]. It has been speculated that these differences between Denmark and Finland may be due to differences in exposures to environmental endocrine disrupting chemicals, especially during sensitive periods of development.

In this study we evaluated the exposure of Danish and Finnish infants to the organochlorine compounds PeCB, HCB, HCH $\left(\alpha-, \beta-, \gamma-, \delta-, \varepsilon_{-}\right)$, DDT-related compounds ( $p, p$ '-DDT, $o, p^{\prime}$-DDT, $p, p$ '-DDD, $o, p^{\prime}-\mathrm{DDD}, p, p$ '-DDE and $o$, $p$ '-DDE), OCS, PCA, aldrin, dieldrin, c-CHL and t-CHL, heptachlor, OXC, cHE, t-HE, methoxychlor (MOC), mirex and END-I by analysing the concentrations of these chemicals in placenta and human milk samples from a prospective birth cohort study. The levels of these compounds in the Finnish placentas have been discussed elsewhere [6]. In addition to looking for geographical differences in the concentrations of these contaminants, we also explored some maternal factors, which have been suggested may affect the prenatal (placenta levels as a proxy) and postnatal (breast milk levels as a proxy) contamination of children.

\section{Method}

Placentas and breast milk samples were derived from a joint prospective, longitudinal birth cohort study in an urban setting in Finland (Turku University Hospital) and Denmark (the University Hospital of Copenhagen) from 1997 to 2001. This study aimed to describe regional prevalence rates and risk factors (lifestyle and exposure) for cryptorchidism by means of questionnaires and biological samples. Recruitment, participation rate, and clinical examination techniques in both countries were completely standardised and described previously [4].

From the total biobank 65 milk samples from each country were included for organohalogen measurements. In Denmark all controls were selected randomly from the entire birth cohort of healthy boys. In Finland the boys were selected prospectively by a case-control design, in which the boys with cryptorchidism were matched to controls at birth for maternal parity, smoking (yes/no), diabetes (yes/no), gestational age ( \pm 7 days) and date of birth ( \pm 14 days). This design was chosen in Finland due to lack of sufficient funding to collect and store biological samples from all. If no matched control milk sample was available a sample from another Finnish control mother was used. Placentas were selected from 112 Finnish mother-child pairs ( $n=56$ boys with cryptorchidism at birth, $\mathrm{n}=56$ controls) and from 168 Danish $(\mathrm{n}=39$ with cryptorchidism, $\mathrm{n}=126$ controls, $\mathrm{n}=3$ boys were first seen at three months of age and were healthy). Funding for placenta analysis allowed including more samples from Danish healthy controls. 86 mother-child pairs (43 Danish and 43 Finnish) were included in with both milk and placenta samples. 
The sample preparation, extraction, cleanup, and high-resolution gas chromatography-high resolution mass spectrometry (HRGC-HRMS) analyses for organohalogen and chiral compounds of the Danish and Finnish samples were carried out in the same laboratory. Briefly, $\mathrm{Na}_{2} \mathrm{SO}_{4}$ (VWR international $\mathrm{GmbH}$, Germany), sea sand (Riedel-de Haen, Germany), alumina B (ICN Biomedical $\mathrm{GmbH}$, Germany), florisil and silica gel (Promochem, UK) were heated at $650^{\circ} \mathrm{C}$ for at least 6 hours before use. Wet samples (10 g placenta tissue or $10 \mathrm{ml} \mathrm{milk})$ were weighed (to a precision of two decimals) and homogenized with $30 \mathrm{~g}$ $\mathrm{Na}_{2} \mathrm{SO}_{4}$ and $15 \mathrm{~g}$ sea sand. Extraction was done in a glass column packed with the homogenized matrix and spiked with ${ }^{13} \mathrm{C}$ internal standards. The extraction solvent was $250 \mathrm{ml}$ acetone and $n$-hexane $(2: 1 \mathrm{v} / \mathrm{v})$. The extracts were collected in flasks weighed in advance and evaporated using a rotary vacuum evaporator (water bath at $45^{\circ} \mathrm{C}$ ). After evaporation, the flasks were placed into a desiccator, until stable weight was achieved. Then the lipid content was calculated on wet weight basis (to a precision of 4 decimals). The lipophilic residual was redissolved in toluene and the clean up was done in gel permeation chromatography (Bio-Beads S-8 column with toluene eluent at $2 \mathrm{ml} / \mathrm{min}$ flow rate) and then in a glass cartridge (packed with alumina $\mathrm{B} 0.8 \mathrm{~g}, \mathrm{Na}_{2} \mathrm{SO}_{4} 0.3 \mathrm{~g}$, florisil $0.5 \mathrm{~g}$, silica gel $1 \mathrm{~g}$, and $\mathrm{Na}_{2} \mathrm{SO}_{4} 0.5 \mathrm{~g}$ from bottom to top). Finally, the sample was condensed to about $10 \mu \mathrm{l}$ and $10 \mu \mathrm{l}$ 1,2,3,4-tetrachlorodibenzo- $p$ dioxin was added as a recovery standard.

The organochlorine compounds were measured by HRGC-HRMS quantified by an isotope dilution method. HP5890 high-resolution gas chromatography equipped with $60 \mathrm{~m}$ DB-XLB column $(0.25 \mathrm{~mm}$ internal diameter $0.25 \mu \mathrm{m}$ film) was used to separate organochlorines. MAT95 high-resolution mass spectrometer was used to detect organochlorines. Three times noise was used as criteria of limit of detection for all data. All other organohalogens were calculated by isotope dilution method.

\section{Result}

There were significant differences between Danish and Finnish mothers in age, parity and smoking habits, with Danish mothers being slightly older, more likely to have a first pregnancy, and more likely to be smoking than Finnish mothers. The BMI of the mothers, the duration of the pregnancy, and the birth weight of the child did not differ between the Danish and Finnish subjects (see Table 1).

Heptachlor, aldrin, $\varepsilon-\mathrm{HCH}$, endosulfan II and t-HE were undetectable in most samples (see Table 2). Most of the other organochlorines were detectable in all samples from both countries. There was no difference between the two countries with respect to the percentage of samples with concentrations above the detection limit for the individual chemicals. $p$, $p$ '-DDE, $\mathrm{HCB}, \beta-\mathrm{HCH}$, dieldrin, $p$, $p$-DDT, END-I, OXC and c-HE were the eight major pollutants detected (together they accounted for $89 \%, 95 \%, 98 \%$ and $98 \%$ of the total pesticide concentration in Finnish placenta, Danish placenta, Finnish milk and Danish milk, respectively). The unadjusted concentrations of these eight chemicals were higher in placentas and milk from Denmark than in samples from Finland. For $p$, 
Table 1: Characteristics of the study subjects.

\begin{tabular}{llll}
\hline & Denmark $\mathrm{n}=190$ & Finland $\mathrm{n}=134$ & $\mathrm{p}$-Value \\
\hline Maternal age (years) & $30.6(23.6-38.8)$ & $28.7(21.4-39.7)$ & 0.011 \\
Maternal BMI $\left(\mathrm{kg} / \mathrm{m}^{2}\right)$ & $22.1(18.0-35.1)$ & $23.0(18.0-37.7)$ & 0.132 \\
Maternal smoking $(\mathrm{n})$ & & & 0.0001 \\
Yes & $64(33.7 \%)$ & $21(15.7 \%)$ & \\
No & $124(65.3 \%)$ & $112(83.6 \%)$ & \\
Parity & $122(64.2 \%)$ & $69(51.5 \%)$ & 0.033 \\
1 & $51(26.8 \%)$ & $44(32.8 \%)$ & \\
2 & $17(8.9 \%)$ & $18(13.4 \%)$ & \\
$\geq 3$ & $282(251-297)$ & $280(256-296)$ & 0.267 \\
Gestational age (days) & $3.6(2.3-4.8)$ & $3.6(2.7-4.5)$ & 0.656 \\
Birth weight (kg) & & & \\
\hline
\end{tabular}

Values are given as medians (range) or numbers. Country differences were tested by the Mann-Whitney U-test.

$p$ '-DDE, $p$, $p$ '-DDT, $\beta$-HCH, HCB, dieldrin, c-HE, and $\mathrm{OXC}$ the levels remained significantly higher in Danish milk samples after adjustment for possible confounders (maternal age, parity, gestational length and date of birth) and for $p$, $p$ '-DDE, $p$, $p$ '-DDT, $\beta$-HCH, HCB, dieldrin, and c-HE the levels remained significantly higher in Danish placenta samples after adjustment.

In a multiple linear regression analysis, lipid concentration, maternal smoking, and maternal BMI did not show any significant correlation to the organochlorine concentrations in milk, whereas maternal age was positively associated and length of pregnancy, infant date of birth, and parity were negatively associated with the concentrations of these eight compounds in milk. Placenta levels of these eight compounds were independent of maternal smoking, but positively associated to maternal age and negatively associated to infant date of birth, and parity. HCB concentration was positively associated with gestational age in placenta. HCB, c-HE, and dieldrin levels in placenta were positively associated with maternal BMI, whereas END-I levels were negatively associated with maternal BMI.

\section{Conclusion}

Our study revealed significant geographical differences in the levels of organochlorine compounds in human placenta and milk samples between two Nordic countries. Danish samples had higher concentrations than Finnish for most compounds. This country difference remained highly significant after adjustment for possible confounders. The findings suggest that despite close vicinity and comparable life styles, exposure may differ significantly between regions. The levels of p,p'-DDE, p,p'-DDT, and HCB in human milk from Sweden, another Nordic country located between Denmark and Finland, have 


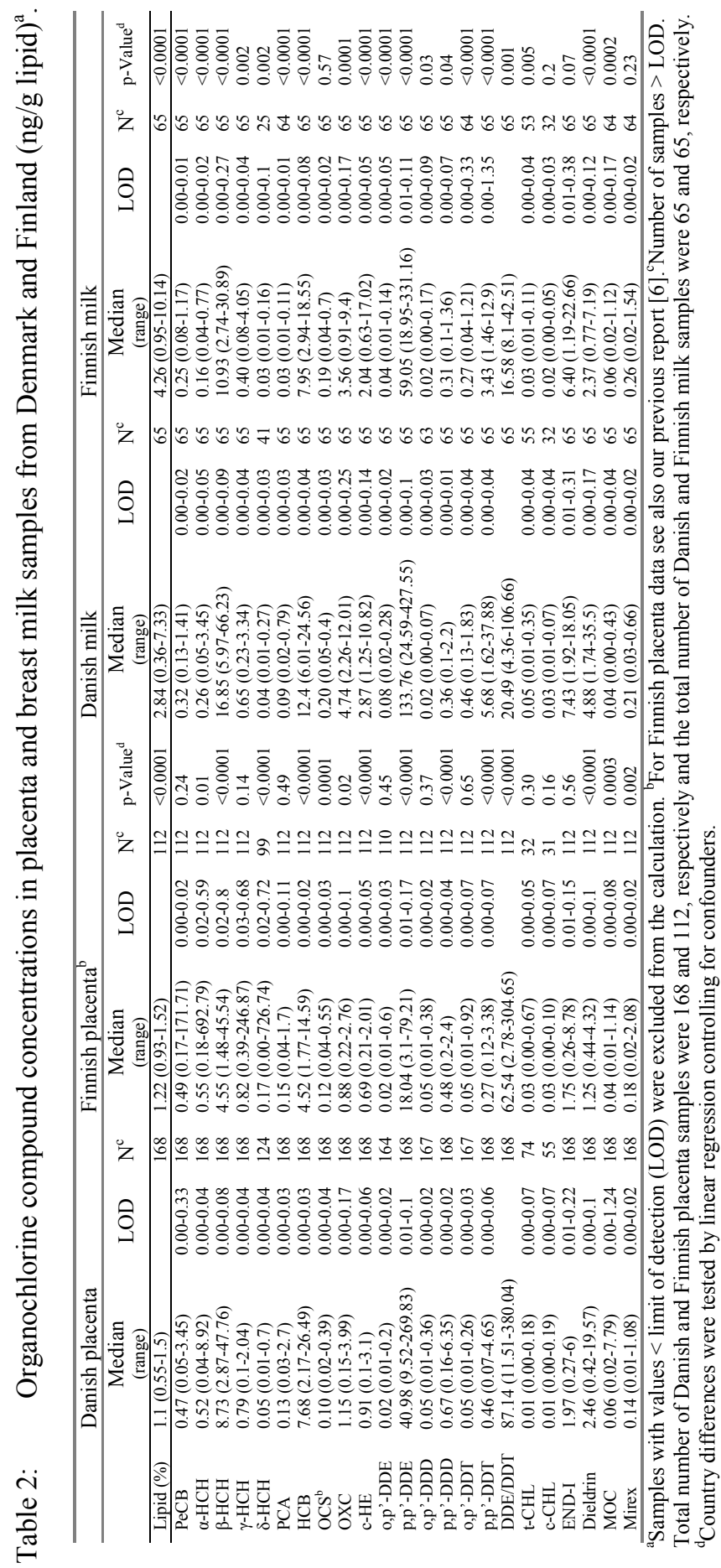


been reported [7]. The levels reported in the Swedish human milk samples (collected in 1997) were comparable to the levels we observed in the Danish milk (collected between 1997-2001).

Several factors, e.g. maternal age, parity, and length of previous lactation have been reported to affect the exposure of the infant [8]. In our study, pollutant levels in human milk and placenta decreased with increasing parity for the majority of the eight most prevalent compounds. This provides additional evidence that previous deliveries and lactation contribute to the clearance of persistent pollutants from the mothers' fatty stores. The concentration of the most prevalent pollutants in milk and placenta were positively correlated to maternal age, which may reflect differences in exposure through dietary habits, metabolism and bioaccumulating properties [9-11].

It has been reported that the clearance rates for DDT, DDE, dieldrin and HCB were $-0.162,-0.113,-0.117$ and -0.118 per year, respectively [7]. We also observed that the levels of many of the measured pollutants were negatively correlated to the date of infant delivery. This finding of slightly decreasing levels of OCs during the brief study period is in line with other reports $[12,13]$.

The correlations between maternal BMI and pollutant levels were not consistent and it remains to be determined whether these associations with maternal BMI are genuine or chance findings due to mass significance.

The observed significant negative correlation of gestational age with the concentrations of c-HE, END-I, and dieldrin in milk samples suggests that a shorter gestational period may lead to a higher mobilization of chemicals stored in maternal fat tissue during breastfeeding instead of during the third trimester during which fetal fat accumulation is greatest. For placenta samples gestational age was only significantly and positively associated with HCB. We suggest that in parallel to the rapid increase of fetal fat stores from around gestational week 20, more pollutants may be released from the mother's adipose depots and transferred to the fetus via placental regulation of fatty acid delivery [14]. Infants born preterm may be less exposed than mature babies to persistent compounds during pregnancy, but then their exposure during breastfeeding may be higher. Thus, the overall exposure appears to be predominantly related to the prenatal maternal body burden. These results should, however, be interpreted with caution as only one of the eight most abundant compounds in placenta correlated with gestational age and thus may also be a chance finding.

The fatty acid composition in placenta is determined by the fatty acid content of maternal liver and plasma [15]. The negative correlation between gestational age and placenta lipid concentration found in our study may be the result of the exponentially increasing fatty acid delivery to the fetus from week 20, which decreases the storage of lipid between the microvillous membrane and the basal membrane of the placenta [14].

In conclusion, multiple factors contribute to the concentrations of chemicals in milk and placenta and thus likely also the pre- and postnatal exposure of infants to persistent organochlorines stored in maternal fat. Our study confirms that maternal age is positively and parity negatively correlated with concentrations of persistent organochlorines. In addition, in this bi-national 
Nordic study we found a significant country difference. This indicates that environmental and lifestyle differences between these two countries exist, which lead to a higher exposure of the Danish population. Recently, an association between congenital cryptorchidism and the combined exposure to the eight most common organochlorines in human milk was indicated [16]. The observed country difference in exposure levels may have been affected by the selection of samples from cryptorchid and healthy boys for this study. The prevalence of cryptorchidism in this subsample is not representative of the true population prevalence [4], neither is the distribution exactly 50:50. However, the exposure difference for organochlorines may play a role for the higher prevalence of male reproductive problems such as testicular cancer, decreased sperm count, and cryptorchidism in Denmark as compared to Finland.

For more detailed information and possible citation of this report please see Hum. Reprod., 2008, 23, 201-210.

\section{References}

[1] Adami H-O, Bergström R, Möhner M, Zatonski W, Storm H, Ekbom A, Tretli S, Teppo L, Ziegler H, Rahu M et al, Testicular cancer in nine Northern European countries. Int J Cancer, 59, pp. 33-38, 1994.

[2] Jørgensen N, Carlsen E, Nermoen I, Punab M, Suominen J, Andersen A-G, Andersson A-M, Haugen TB, Horte A, Jensen TK et al, East-West gradient in semen quality in the Nordic-Baltic area: a study of men from the general population in Denmark, Norway, Estonia and Finland. Hum Reprod, 17, pp. 2199-2208, 2002.

[3] Virtanen HE, Kaleva M, Haavisto A-M, Schmidt IM, Chellakooty M, Main KM, Skakkebæk NE, and Toppari J, The birth rate of hypospadias in the Turku area in Finland. APMIS,109, pp. 96-100, 2001.

[4] Boisen KA, Kaleva M, Main KM, Virtanen HE, Haavisto A-M, Schmidt IM, Chellakooty M, Damgaard IN, Mau C, Reunanen M et al, Difference in prevalence of congenital cryptorchidism in infants between two Nordic countries. Lancet, 363, pp. 1264-1269, 2004.

[5] Boisen K, Chellakooty M, Schmidt I, Kai C, Damgaard I, Suomi AM, Toppari J, Skakkebæk NE, and Main K, Hypospadias in a cohort of 1072 Danish newborn boys: Prevalence and relationship to placental weight, anthropometrical measurements at birth, and reproductive hormone levels at 3 months of age. J Clin Endocrinol Metab, 90, pp. 4041-4046, 2005.

[6] Shen H, Main KM, Kaleva M, Virtanen HE, Haavisto A-M, Skakkebæk NE, Toppari J, and Schramm KW, Prenatal organochlorine pesticides in placentas from Finland: exposure of male infants born during 1997-2001. Placenta, 26, pp. 512-514, 2005.

[7] Noren K and Meironyte D, Certain organochlorine and organobromine contaminants in Swedish human milk in perspective of past 20-30 years. Chemosphere, 40, pp. 1111-1123, 2000. 
[8] Harris CA, Woolridge MW, and Hay AW, Factors affecting the transfer of organochlorine pesticide residues to breastmilk. Chemosphere, 43, pp. 243256, 2001.

[9] Brunetto R, Leon A, Burguera JL, and Burguera M, Levels of DDT residues in human milk of Venezuelan women from various rural populations. Sci Total Environ, 186, pp. 203-207, 1996.

[10] Covaci A, Jorens P, Jacquemyn Y, and Schepens P, Distribution of PCBs and organochlorine pesticides in umbilical cord and maternal serum. Sci Total Environ, 298, pp. 45-53, 2002.

[11] Czaja K, Ludwicki JK, Goralczyk K, and Strucinski P, Effect of age and number of deliveries on mean concentration of organochlorine compounds in human breast milk in Poland. Bull Environ Contam Toxicol, 59, pp. 407413, 1997.

[12] Solomon GM and Weiss PM, Chemical contaminants in breast milk: time trends and regional variability. Environ Health Perspect, 110, pp. A339A347, 2002.

[13] Smith D, Worldwide trends in DDT levels in human breast milk. Int $J$ Epidemiol, 28, pp. 179-188, 1999.

[14] Haggarty P, Placental regulation of fatty acid delivery and its effect on fetal growth--a review. Placenta, 23 Suppl A, pp. S28-S38, 2002.

[15] Graham CB, Rebecca LD, and Alan AJ, The effect of reduced maternal protein intake during pregnancy on placental lipid composition in the rat: effect of glycine supplementation of the low protein diet. Nutrition Research, 24, pp. 909-921, 2004.

[16] Damgaard IN, Skakkebaek NE, Toppari J, Virtanen HE, Shen H, Schramm KW, Petersen JH, Jensen TK, The Nordic Cryptorchidism Study Group, and Main KM, Persistent pesticides in human breast milk and cryptorchidism. Environ Health Perspect, 114, pp. 1133-1138, 2006. 